\section{Viability of Stored Bone Marrow Colony Forming Units}

THE continued use of bone marrow transplantation ${ }^{1}$ in disease states and aftcr accidental or therapeutic exposure to radiation has led to an increasing number of in vitro and in vivo studies of the viability of human and animal bone marrow. Among the more recent methods for quantitative studies of animal marrow is the mouso spleen colony technique. Till and McCulloch ${ }^{2}$ were the first to show that syngeneic mouse bone marrow, when injocted into animals which had received a lethal dose of radiation, will cause the formation of macroscopic spleen nodules in a number directly proportional to the number of injected cells. These discrete colonies are assumed to arise from single marrow cells, which are generally described as colony forming units (CFU) and are often equated with the marrow stem cell. This method of studying colony formation has been used to study stem cell kineties ${ }^{3}$, cellular radiation sensitivity ${ }^{2}$, immune reactions, anaemic mutants ${ }^{5}$, and various phases of haematopoietic repopulation in the normal and postirradiation states.

In spite of the wide use of these techniques in studies which involve a delay between collection of the marrow cells and reinjection, little attention has been given to the effect of temperature, storage and suspending media on the viability of CFUs. A series of determinations was therefore made to evaluate the viability of the bone marrow CFU after suspension in two common suspending media at room and ice temperatures. The suspending

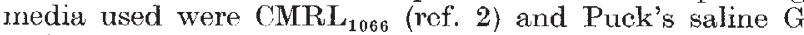
(ref. 6), which is a buffered nutrient saline mixture. For cach determination, primary cell suspensions were obtained by collecting the marrow colls from the femurs and tibiae of two donor mice. In all cases donor and recipient mice wore 8 week old white males of the Hale-Stoner Brookhaven struin. The bone marrow colls were collected by the Stoner and Bond method?. This involved thorough grinding with a pestle and mortar of the entiro fomur and tibia in a small amount of tho suspending medium. This method not only shortened the preparation time but also gave a higher yield of CFUs than did tho aspiration technique in which portions of the femur and the entire tibia were not used. After the two primary cell solutions had been obtained they wero divided into equal portions

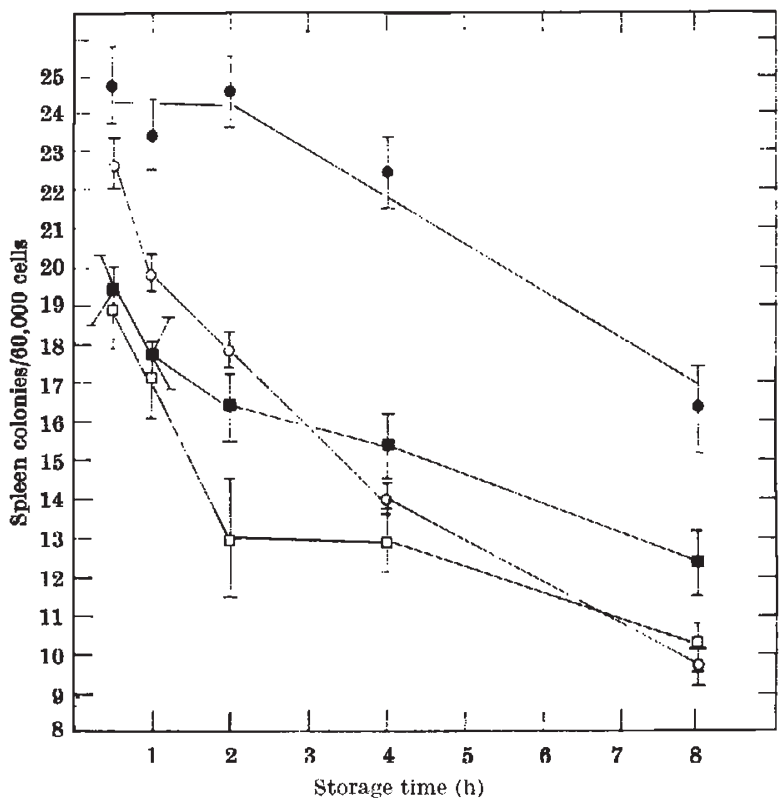
Fig. 1. Viability of stored colony forming units. - Saline $G$, ice ice temperature; $\square$ - CMKL with one portion of each being maintained at room temperature while the other was placed in an ice bath. The cellular content of the solutions was determined by counting an aliquot treated with saponin in a Coulter counter with a $100 \mu$ aperture. Using the proper medium, the standard solutions were then diluted to a concentration of $6 \times 10^{5}$ cells $/ \mathrm{ml}$. At the time intervals indicated, $1 / 10$ ml. $(60,000$ cells) were injected, via the tail vein, into each animal which had received a whole body exposure of 700 rads of $250 \mathrm{kVp} \mathrm{X}$-rays during the hour before the injection. After 7 days the recipient animals were killed, and their spleens placed in Bouin's solution for $24 \mathrm{~h}$, after which the macroscopic colonies were counted using a times three magnifier. Three replications of the entire procedure were carried out to produce six donor and twenty-five recipient animals at each of the points indicated in Fig. 1 .

Two facts are immediately apparent from the data expressed in Fig. 1. First, maintenance of cell solutions at the temperature of ice preserves their viability for longer periods than does maintenance at room temperature. Second, viability of the cells depends on which of the two media is used. The saline $G$ medium can maintain cells for longer and the most marked differences between the media appear at the shortest time interval $(30 \mathrm{~min})$. This early effect suggests that there is a sub-population of colls which are immediately rendered non-viable in the CMRL $_{1068}$ solution.

Wide variations in CFU content of the mouse marrow have been reported, and by determinations on three different mouse strains we have shown strain dependence to be a factor. Our results also show that some of the variation in the officiency of marrow to form colonies may be due to such simple factors as suspending medium, temperature, and delay between the harvesting of marrow and the injection of cells.

The close control of handling techniques may be even more important when investigating the type as well as the number of colonies formed. The histological nature of spleen colonies has been studied quantitatively8-10. The difference in type of resultant colony is usually attributed to either a different type of original colony forming unit or to the microenvironment in which the injected cell finds itself. If the original cell determined the later colony type, consideration must be given to the possible selective sensitivity of the original cell types to the suspending media, temperatures, storage time and so on. We are now investigating the extent to which the ratio of myelocytic to erythrocytic colonies might be altered by these factors.

This research was supported by the US Atomic Energy Commission. We thank Jim Cassidy, Larry Cook and Keith Thompson for their assistance in the technical and statistical aspects of the study.

A. L. Carsten V. P. BOND

Medical Research Center,

Brookhaven National Laboratory,

Upton, Long Island,

New York.

Received June 17, 1968.

${ }^{1}$ Pegg, D. E., in Bone Marrow Transplantation (Lloyd-Luke Ltd, London, 1966)

2 Till, . F. F., and MeCulloch, E. A., Radiat. Res., 14, 213 (1901).

${ }^{3}$ Till, J. F., MoCulloch, E. A., and Siminovitch, L., Proc. US Nat. Acad. Sci., $51,29(1964)$

${ }^{4}$ Trentin, J. T., and Fahlberg, W. J., in Conceptual Advances in Immunology and Oncology, 66 (Harper (Hoeber), New York, 1963).

MeCulloch, E. A., Siminoviteh, L., and Till, J. F., Seienee, 144, 844 (1964).

- Puck, T. T., Cieciura, S. J., and Robinson, A., J. Exp. Med., 108, 945 (1958).

"Stoner, R. D., and Bond, V. P., Exp. Hematol., 91 (2), 185 (1963).

${ }^{8}$ Curry, J. L., and Trentin, J. J., Dev. Biol., 15 (5), 395 (1967).

'Lewis, J. P., O'Grady, Lois F., and Trobaugh, jun., F. E., Exp. Hematol., 12, 33 (1967).

${ }^{10}$ Wolf, N. S., and Trentin, J. J., J. Fxp. Med.,127 (1), 205 (1988). 